\title{
On the quality of knowledge in the field of electricity and magnetism
}

\author{
Monica G. M. Ferguson-Hessler and Ton de Jong \\ Department of Physics and Department of Philosophy and Social Sciences, Eindhoven University of \\ Technology, Eindhoven, The Netherlands
}

(Received 9 January 1986; accepted for publication 6 August 1986)

\begin{abstract}
Problem solving in physics requires a certain quantity of knowledge of the subject matter: principles, procedures, etc. In addition, the problem solver must be able to access these principles and procedures in a given situation. Investigations have shown that failure in problem solving is often caused by lack of availability of knowledge, and also that availability is closely related to the organization of knowledge in memory. Opinions differ, however, on whether the optimal form of this organization should be centered around problem types or arranged in a hierarchical way. In this study two concrete examples of knowledge structures in the field of electricity and magnetism are compared. An experiment is also described, in which the actual knowledge structure of beginning students was studied. The outcome indicates that students with good results in problem solving organize their knowledge more in accordance with problem types than do students with poor results. The results of the experiment are discussed in the light of the two knowledge structures described. The possible role of these structures in physics teaching is treated in the final paragraph.
\end{abstract}

\begin{abstract}
"Physicists often claim that the great beauty of Physics (unlike some other fields, such as organic Chemistry) is that there is relatively little to remember. By contrast many novice students complain that there is so much to remember. Both are probably right." (Reif, ${ }^{1}$ p. 47)
\end{abstract}

\section{INTRODUCTION}

The teaching goals of an undergraduate physics course are usually expressed in terms like "insight into" and "understanding of." In this way a quantity of knowledge is defined, a domain of the subject that has to be mastered. Sometimes the goals are worked out explicitly in objectives like "being able to define..." or "being able to apply... in... ." Here the quality of this knowledge is defined: The knowledge should be applicable in certain, specified types of situations. The tests used at many universities to assess students' attainments are often made up of problems, where knowledge has to be applied in new situations, not merely reproduced. In other words: the capacity for solving problems, is seen as a measure of the quantity as well as of the quality of the knowledge acquired. In this study we concentrate on the quality of knowledge of physics in an attempt to demonstrate the explicit meaning of this concept for a particular field at a particular level: Electricity and magnetism at the level of a first year undergraduate course.

\section{SOME CURRENT THEORIES ON THE ORGANIZATION OF KNOWLEDGE IN DOMAINS LIKE PHYSICS AND MATHEMATICS}

It is well known by teachers that many students do not succeed in solving physics problems in spite of the fact that they are able to reproduce an appreciable amount of knowledge of formulas and definitions. On the other hand, in an investigation of the reasons for failure in solving problems in electricity and magnetism (E\&M) ${ }^{2}$ we found indications that one of the shortcomings which play an important role is lack of availability of knowledge, i.e., not being able to "find" the principles and procedures needed.
This sort of knowledge, which can be reproduced when directly asked for but which is not available when needed in problem solving, demonstrates the need of a concept like quality of knowledge.

In this study we use the concept of quality to denote the applicability of knowledge in new situations, e.g., in problem solving and experimental work.

According to this definition availability is an important aspect of the quality of knowledge. Most authors investigating problem solving in physics and mathematics (e.g., Refs. 1,3-6) agree that an adequate organization of knowledge in memory will make this knowledge more easily available when it is needed for problem solving. As to the explicit form of "an adequate organization" there is less agreement. Before entering into this discussion it is necessary to look a bit closer at the concept of knowledge of physics itself, as it applies to the content of an undergraduate course.

\section{A. Types of knowledge}

In discussions on the role of knowledge in problem solving it is useful to distinguish between knowledge that is bound to a certain subject and knowledge that can be transferred to other subjects. Knowledge of strategy can be applied in a variety of subjects if it is formulated in general terms, such as the four phases described by Reif et al. ${ }^{3}$ : "description," "planning," "implementation," and "checking." Consequently strategy is not included in the structures discussed in this study. Subject knowledge is usually divided into declarative knowledge, consisting of definitions, principles and theorems, formulas, facts, etc. and procedural knowledge, comprising the "rules of the game": how to apply a certain formula, e.g., how to choose a Gaussian surface.

In prior studies ${ }^{7}$ we have found it useful to distinguish a third type of subject knowledge, that of problem situations, i.e., characteristics of a given situation which are relevant for the selection of solution principles. Formally this form of knowledge could be classified as declarative, but its function in problem solving is different from the function of 
formulas and laws. It serves to extract from the more or less implicit description of the given situation those characteristics which can serve as cues for the search in memory for relevant and useful formulas and procedures. These cues are what Chi et al. ${ }^{6}$ call "second-order features."

The elements of these various types of knowledge are not stored independently in memory but are related to each other, forming a "knowledge structure." The form and usefulness of these structures are the subject of the following paragraphs.

\section{B. Problem-oriented knowledge structures}

Problem solving in physics, especially in mechanics, has been studied by Larkin, ${ }^{8-10}$ Reif, $^{3} \mathrm{Chi},{ }^{6}$ and others. These researchers compared the performance of novice students to that of experienced university teachers, experts. Great differences were found in solution strategies and in classification of problems. These differences were explained in terms of knowledge structures: Experts have their knowledge organized in "chunks" or "schemata," i.e., elements of knowledge which are closely related and often jointly used are connected in a cluster. Novices have a much less outspoken organization of their knowledge, although the number of elements of knowledge of a certain field in their memory is not necessarily smaller than that of the experts.

The concept of "problem schema," which has become widely used in discussions on problem solving, can be defined as follows: A problem schema consists of a limited number of elements of knowledge, all related to a central law or concept, which is used in solving a certain category of problems. An adequate schema contains all the subject knowledge needed for the solution, i.e., declarative and procedural as well as situational knowledge.

\section{The hierarchical organization of knowledge}

A detailed analysis of the process or problem solving in physics has been carried out by Reif. ${ }^{1,11}$ One of the most important stages of this process is the search in memory for elements of knowledge which are relevant and useful in the given situation. Reif states that an efficient search process is greatly facilitated if the knowledge present in memory is organized according to a principle of hierarchy. This means that knowledge of a given subject is described at different levels of detail and abstraction.

As an example Reif and Heller ${ }^{11}$ have constructed a knowledge structure for mechanics. At the highest level of abstraction the complete knowledge of this subject is represented by four concepts, called "individual descriptors," "interaction descriptors," "laws of interaction," and "principles of motion." This level specifies all the types of entities used and their function, i.e., it contains a metacognitive description of the subject. On the second and lower levels each of the four concepts is successively specified and split up into more detailed concepts like the "motion descriptors" of a particle: "position," "velocity," and "acceleration."

The relationship between the two types of knowledge structures will be discussed in Sec. III C.

AMPERE' S LAW.

Elements of declarative knowledge.

1. Ampère's law.

2. $\phi \overrightarrow{\mathrm{B}} \cdot \mathrm{d} \overrightarrow{\mathrm{S}}=\mu_{0} \mathrm{I}_{\text {enclosed }}$

3. Closed path p.

4. Current enclosed by path p.

5. Right hand screw rule.

\section{Elements of procedural knowledge.}

6. Analyzing the symmetry properties of a given current distribution.

7. Choosing a closed path $\mathrm{p}$, along which the line integral

$\oint \vec{B} . d \vec{s}$ can be conveniently calculated.

8. Choosing a closed path, enclosing an element of surface current.

Fig. 1. A problem scheme.

9. Deciding on the direction in which to calculate the line integral.

10. Choosing a suitable surface for the calculation of Ienclosed.

11. Calculating $\iint \vec{J} \cdot d \vec{A}$ over this surface.

Characteristics of problem situations.

12. Long straight current.

13. Plane, homogeneous surface current density.

14. Space current density with cylindrical symmetry.

15. Cylindrical surface current.

16. Magnetic field with cylindrical symmetry.

17. Long solenoid.

18. Toroid. 


\section{EXAMPLES OF KNOWLEDGE STRUCTURES IN THE FIELD OF ELECTRICITY AND MAGNETISM}

What is the role of the various knowledge structures in problem solving in the field of electricity and magnetism, and what is the role they can play in teaching? In search for an answer to these questions we worked out a set of problem schemata covering a first course on E\&M and also a hierarchical knowledge structure of the field. The results and conclusions are discussed below.

\section{A. The problem schemata}

As we were especially interested in the knowledge of novice students, we analyzed the contents of the first-year course E\&M I and structured it in the form of schemata. Each schema is centered around a fundamental law or concept and contains elements of declarative knowledge as well as procedures and characteristics of problem situations.

The course covers the following main topics:

electrostatic field in vacuum,

magnetostatic field in vacuum,

the Lorentz force,

electromagnetic induction.

Twelve different types of problems were distinguished within these topics, each related to a law, like "Ampère's law," or to a fundamental concept, like "capacitor" or "potential."

For each problem type a complete schema was constructed. An example of such a schema is given in Fig. 1. It contains the concepts, definitions and formulas related to the problem type, i.e., the declarative knowledge needed to solve this type of problem (elements 1-5). It also contains the procedures necessary in applying the declarative knowledge (elements 6-11), and the characteristics of problem situations in which this knowledge can be applied in a useful way (elements 12-18). In the actual case, e.g., it is important to know that Ampère's law is useful for a long solenoid but not for a single, circular, current loop.

Each schema thus contains all the main elements of knowledge which are necessary in order to analyze the given situation, map out the solution and carry this out. Ancillary knowledge, like vector algebra and integration has not been included in the schemata.

\section{B. A hierarchical structure}

The first conclusion in trying to construct an example of a hierarchical knowledge structure covering the field of electricity and magnetism is that this subject matter is intrinsically hierarchic. However, this becomes evident only when the whole of classical electromagnetism is included in the structure.

Two fundamental concepts require adequate "descriptors" in electromagnetism: fields and matter. Their interactions are of three different types: field-field (e.g., electromagnetic induction), matter-field (e.g., matter as a source of fields), and field-matter (e.g., polarization). Mattermatter interaction is considered as an "interaction-at-adistance," mediated by the field. The first two types of interaction are described in the Maxwell equations which give a complete account of the behavior of the electromagnetic field under influence of matter. Field-matter interac-

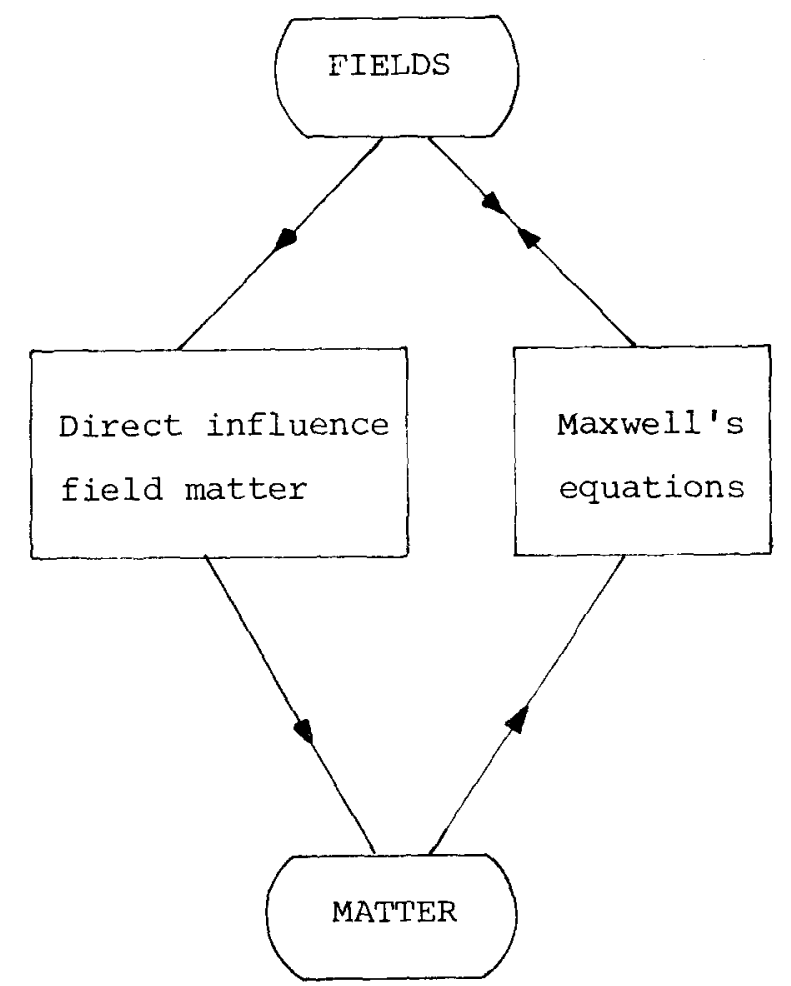

Fig. 2. The highest level of the hierarchical knowledge structure.

tion is described by a separate set of relations. This metacognitive description of electromagnetism is illustrated in Fig. 2, which pictures the highest level of a hierarchical knowledge structure of this subject.

In the second level of this structure specification and diversification is introduced. The concept of "field" has a number of different aspects like field vector, flux, energy density, principle of superposition, and vector potential. Each of these is further specified and stated in explicit form in the third level of the structure, e.g., the fundamental vector fields $\mathbf{E}(\mathbf{r}, t)$ and $\mathbf{B}(\mathbf{r}, t)$ as well as their alternative field descriptors $\mathbf{A}(\mathbf{r}, t)$ and $\varphi(\mathbf{r}, t)$ and the relations of $\mathbf{A}$ and $\varphi$ to the vector fields.

Another example of the second level is the specification of the Maxwell equations given in the upper part of Fig. 3. The various types of interaction mentioned above are described by separate terms of the differential equations, e.g., the right-hand terms of the first Maxwell equation describe the interaction of $\mathbf{H}$ with $\mathbf{D}$ and $\mathbf{J}$, respectively.

For further specifications at the third level the Maxwell equations are written in their integral form. In the quasistatic approximation $(\partial \mathbf{D} / \partial t \approx 0)$ the influence of material sources is seen to become isolated in the well-known laws of Ampère and Gauss, while the field-field interaction is described by Faraday's law. The third equation indicates the absence of magnetic charges as a source for the B field. Also the experimental laws forming the basis of the first and fourth Maxwell equation appear at this level.

Some of the formulas forming the central part of the problem schemata of Sec. III A appear here as part of the third level of the hierarchical structure. In the same way all the "kernels" of the schemata are seen to form part of one of the lower levels of the hierarchical structure. 


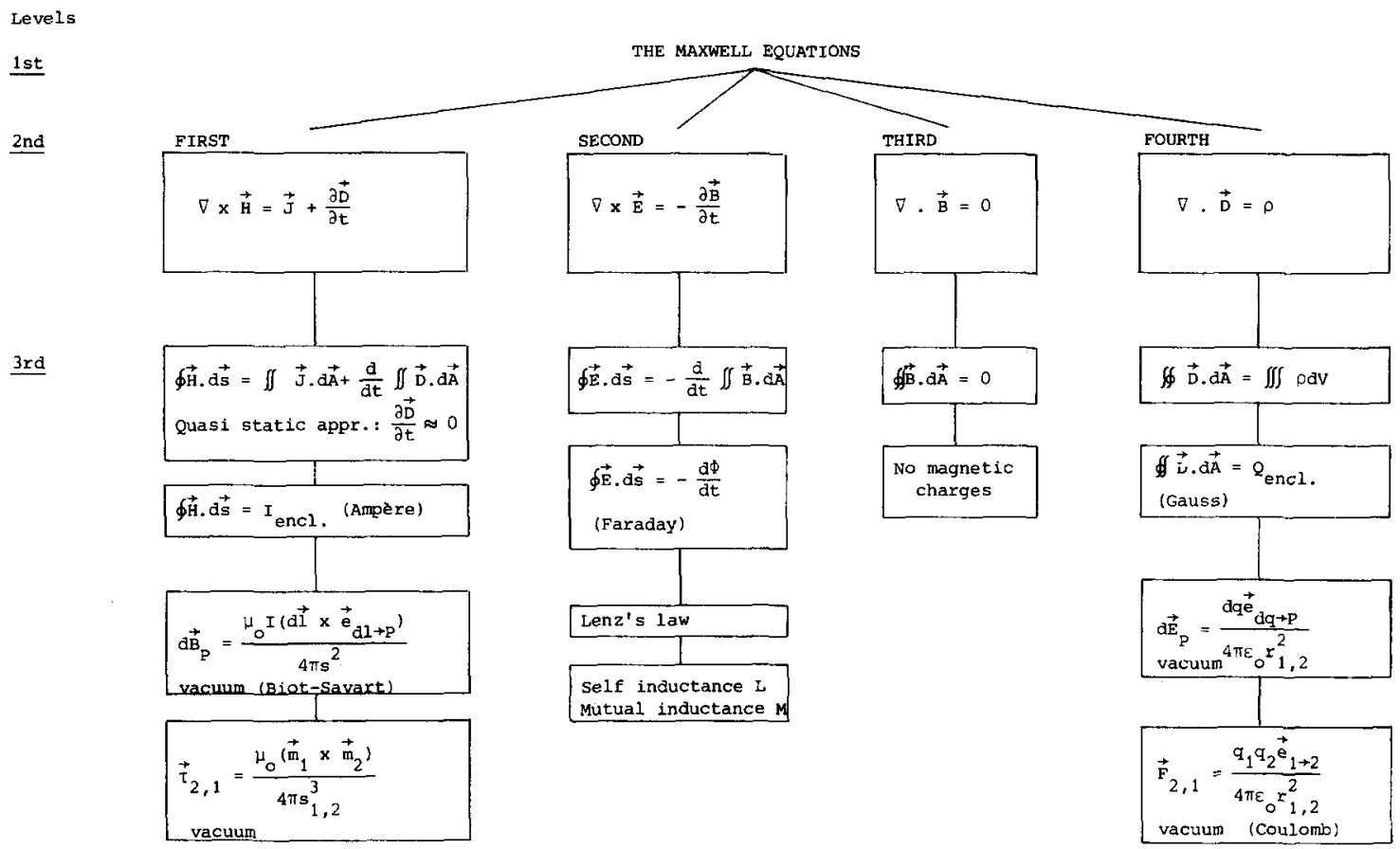

Fig. 3. The Maxwell equations as part of a hierarchical knowledge structure.

\section{A comparison of the two types of knowledge structure}

When comparing the hierarchical organization of knowledge of electromagnetism with the system of schemata of Sec. III A, we were lead to the following conclusions:

(1) The problem schemata, centered around formulas like Ampère's law or around a fundamental concept like potential, can be fitted into the hierarchical structure to form the fourth and lowest level.

(2) It is not possible to build a hierarchical knowledge structure from the elements of knowledge contained in the problem schemata. The more abstract and more general concepts which form the higher levels of such a structure are not included in the problem schemata depicting the contents of a first course on the subject.

(3) The knowledge contained in the hierarchical knowledge structure cannot be harbored in a set of problem schemata.

It seems that the two types of organization of knowledge of electromagnetism do not contradict but supplement each other: At the level of a first course, where the possibilities for abstraction are limited, problem schemata form an efficient means of organizing the elements of knowledge, thus making them available for problem solving. As the amount of knowledge grows, new concepts are learned which are of a more abstract and general nature, and new connections are made between concepts already known. This makes it possible to organize the problem schemata into a structure with increasingly hierarchical features which, in the end, will cover all the central issues of classical electromagnetism. Once this has been achieved, one of the advantages of a hierarchical structure (Reif ${ }^{\mathrm{l}}$ ) is demonstrated: New areas of the subject can be fitted into the structure without major changes, e.g., the theory of electromagnetic radiation or plasma physics.

This discussion has implications for teaching which will be treated in Sec. V.

\section{AN EXPERIMENT ON THE USE OF KNOWLEDGE STRUCTURES BY FIRST YEAR STUDENTS}

Inspired by the experiments and theories described in Sec. II we decided to investigate if some aspects of the differences between experts and novices, found by several authors, would also exist between novice students with good and poor performance in problem solving. An experiment was set up in order to study the knowledge structures in the field of electricity and magnetism of first year students and to test the following hypothesis:

Good novice problem solvers have their knowledge organized according to problem types, whereas poor novice problem solvers lack this organization.

This required four steps:

(1) defining an "ideal" organization of knowledge, a structure centered around types of problems.

(2) studying the actual knowledge structure of a number of novice students.

(3) developing a quantitative measure of the agreement between the students' structure and the ideal one.

(4) correlating this measure to a measure of success in problem solving.

This experiment is described in Ref. 7. Below we give a brief summary and then concentrate on the results, which are of importance for the discussion on teaching.

The "ideal structure" was defined as the set of problem schemata of Sec. III A. The knowledge structure of a number (47) of students was studied by means of a card sorting technique. Elements of knowledge from the problem schemata were printed on separate cards and each student was given the deck of cards (65 in total) and asked to sort the cards in piles in such a way that "cards on the same pile were more strongly connected to one another than to the cards of other piles." A numerical measure was defined for the agreement between the organization of the knowledge 
elements according to the piles of each student and the problem schemata. This measure was correlated with the marks on the problem solving examination on electricity and magnetism, which had taken place about a week before the experiment.

The coefficients of correlation found were up to 0.54 $(p<0.01)$, quite a high value, taking into account the many different factors that influence examination results. This indicates that students with high marks do indeed have their knowledge organized according to problem schemata. The result was confirmed by analysis of the data with a different technique which does not deal with the results of individual students but which compares the clusters typical of two groups of students. A hierarchical cluster analysis was performed of the piles of cards produced by two groups of students, one with high marks $(\geqslant 7$ on a 10 point scale) and one with low marks ( 2 or 3 ). The typical clusters of the two groups resulting from the analysis are quite different: clusters typical of good students agree with the problem schemata in most aspects, while clusters typical of poor students show little agreement with the schemata.

Also an analysis was performed of the labels, which the students had been asked to give the piles. These labels were compared to the contents of the piles in an attempt to discover the types of criteria that had been used in sorting. Clear indications were found that good problem solvers use mainly criteria that are relevant to the physics contents of the knowledge elements, like "related to induction," whereas poor problem solvers tend to use superficial criteria, like "containing the word field."

A striking result in this experiment was the small number of elements of knowledge put aside as "unknown," not more that $2 \%$ for good and $4 \%$ for poor problem solvers. Thus, even students with very low marks learned the individual elements of knowledge.

The results confirm that good novice problem solvers organize their knowledge more in accordance with problem types than poor novice problem solvers do. Also, in the criteria used in sorting we recognize the "deep structure" and "surface features" found by Chi et al.

\section{ON THE ROLE OF KNOWLEDGE STRUCTURES IN TEACHING PHYSICS}

Several experiments (de Groot, ${ }^{12}$ Larkin, ${ }^{5}$ Chi et al. ${ }^{6}$ ) have shown that the difference between expert knowledge and novice knowledge is essentially a matter of organization, or structuring. Our card sorting experiment indicates that this sort of difference also exists between successful and unsuccesful students: They are distinguished by their way of sorting, not by the number of elements known.

The results, however, are purely correlational and, as such, do not give any information on the cause of the differences in examination results. Still, comparing our results to those of other researchers like Larkin ${ }^{5}$ and Chi et al. ${ }^{6}$ on the importance of "chunks" and "problem schemata," we think they warrant the conclusion that quality of knowledge is closely related to the way in which it is organized.

This has consequences for teaching physics. Most textbooks present the contents of the subject in a linear way and give little attention to its organization. Many students do not succeed in organizing their knowledge of physics in a meaningful way. How can teaching staff focus attention on the role of the structure of knowledge in problem solving, and how can we stimulate students to improve the quality of their knowledge?

\section{A. Which type of organization}

It is a general experience that students, when given a problem to solve, tend to start on calculations without first analyzing the situation. A number of researchers (Larkin and Reif, ${ }^{10}$ Mettes, Pilot and Roosink, ${ }^{13}$ Bruce and Heikkinen, ${ }^{14}$ and Ferguson-Hessler and de Jong ${ }^{2}$ among others) have tried to teach explicitly how to analyze problem situations, but with varying success. Students seem to prefer sticking to the method of "kick and rush": get hold of a formula, calculate, ready! In other words, students act as if they had a problem schema which is relevant in the given situation, although this is often not the case.

It seems that making explicit use of problem schemata in teaching would be in agreement with this tendency of students and also, what is more important, with the results of several studies indicating that problem schemata are useful for solving problems at the level of a first year course. In this way one could hope to stimulate students to learn to recognize a number of standard problem situations and to recall the laws and procedures which are relevant in the given situation.

But problem schemata have more content than standard problems with standard solutions. They provide a framework for the organization of elements of knowledge which are closely related and often jointly used, but which are learned at different moments and in different ways, like definitions of concepts used, conditions of validity, and symmetry properties of problem situations. Therefore problem schemata are a useful tool for analysis: They tell the solver what sort of information to look for, help to link this information to knowledge elements in long-term memory, and also suggest possible subproblems for the solution. Also these schemata are helpful in problem situations where not all the necessary information is given, but part of it has to be collected by the solver.

On the other hand the organization of knowledge in problem schemata is incomplete in the sense that relations between the laws and principles constituting the "kernels" of the schemata are not integrated into the structure. This can be remedied by explaining the relations between the schemata as far as this is possible within the limits of the subject matter. In practice these limits are fairly narrow for most introductory courses on E\&M. In more advanced courses on electromagnetism (note the difference of name!) the abstract and general concepts are introduced which make it possible to build a hierarchical knowledge structure where the schemata fit in as building blocks at the lower levels.

There are indications (Reif," de Jong and FergusonHessler $^{7}$ ) that experts organize their knowledge in this way. Such a knowledge organization reduces the load on long-term memory, makes the various elements of knowledge easily available, and may well lead to the experience "there is relatively little to remember" (Reif').

Acquiring such a knowledge structure can be considered as the long-term goal of the study of a subject like classical electromagnetism. 


\section{B. Means and possibilities of teaching the structure of knowledge}

Last paragraph sketched a goal of learning, a hierarchical knowledge structure, growing out of an initial set of problem schemata. Here we discuss some possibilities of teaching the structure of subject matter.

One could argue that knowledge structures should be explicitly taught in the same way as the knowledge content, or, on the contrary that an implicit way of teaching is preferable, as students have to discover the structures themselves. The solution of this dilemma may lie one level deeper: To teach, or at least to demonstrate, how to build up a knowledge structure of your own and how to apply this structure in problem solving.

One way of doing this is to describe explicitly of the relations used, the associations followed, and the visualizations created in solving a problem or analyzing a theory. Some of the difficulties students experience in learning problem solving in physics are caused by the fact that lecturers and instructors often handle their own knowledge structures in a very implicit way. In working out solutions they use an appreciable amount of tacit knowledge ( Reif $^{1}$ ). The reason is perhaps that often lecturers are not aware of the differences between their own, probably highly structured, organization of knowledge and the less systematic knowledge structures of students. These differences may be one of the reasons that some students find it difficult to follow the application of knowledge demonstrated in problem solving classes. They faithfully copy everything down but are not capable of joining a discussion on the solution demonstrated.

Explicit use of problem schemata of the type described in Sec. III A will help to make the tacit knowledge of the teacher visible to the students. In this way attention may be given to the characteristics of the problem situation and the way in which these lead to the choice of one or more specific principles for the solution, and to the rejection of other possible principles.

When the quantity of knowledge grows and its structure becomes more complicated, it is useful if the schemata are well established in memory and can be used as a whole without detailed specifications. This will considerably reduce the load on working memory, especially in problem solving, and free attention for deeper analysis and planning.

As to the practical means of stimulating students to build up their knowledge structure one possibility is to hand out ready-made schemata and overviews. However, in the opinion of some experienced teachers of physics (e.g., Arons ${ }^{15-18}$ ) only active reasoning of his/her own can help the student to real understanding of physical concepts and relations:

"Didactic explanation and a concentrated remedial exercise do not help the majority of students to overcome a cognitive difficulty. Much greater success is achieved through providing students with repeated opportunity, in slightly differing situations, to trace the line of reasoning and articulate it in their own words, either orally or in writing."'15

The own reasoning of students can be stimulated by letting the structure of a certain part of the subject grow on the blackboard in the course of a discussion between lecturer and students or in a group of students, and then leaving it to the individual student to work out the results more in detail.

At the Eindhoven University of Technology the students taking examinations in physics subjects in the first years are stimulated to make an overview of the subject in a different way: They are allowed to bring along a limited amount of personal notes on the subject. In constructing the examination problems and the marking schemes we take this into account. Merely writing down a formula (which could be copied from the notes) will not lead to credit points; the student must show its relation to the given situation or how it is to be used. We stress the importance of the working out of these notes as an essential part of the study of the subject. Alas, the notes produced are often more a result of trying to cram a maximum amount of writing into the number of pages allowed than of interrelating elements of knowledge and building up a structure. Thus they demonstrate to the lecturer that she has not succeeded in teaching the subject in such a way that most students reach the point where "there is relatively little to remember" (Reif').

\section{ACKNOWLEDGMENTS}

We want to thank Professor W. Vaags, Professor G. J. Mellenbergh, and Dr. C. Hamaker for helpful comments and discussions in the course of the experiment; and Professor W. van Haeringen, Professor F. de Hoog, and Dr. G. Nijgh for interesting and clarifying discussions on the physics.

${ }^{1}$ F. Reif, in Research on Physics Education (Centre National de la Recherche Scientifique, Paris, 1984).

${ }^{2}$ M. G. M. Ferguson-Hessler and T. de Jong, in Research on Physics Education (Centre National de la Recherche Scientifique, Paris, 1984).

${ }^{3}$ F. Reif, J. H. Larkin, and G. C. Brackett, Am. J. Phys. 44, 212 (1976).

${ }^{4} J$ J. G. Greeno, Ed. Psychol. 12, 262 (1978).

${ }^{5}$ J. H. Larkin, Science 208, 1335 (1980).

${ }^{6}$ M. T. Chi, P. J. Feltovich, and R. Glaser, Cognitive Sci. 5, 121 (1981).

${ }^{7}$ T. de Jong and M. G. M. Ferguson-Hessler, J. Ed. Psychol. 78, 279 (1986).

${ }^{8}$ J. H. Larkin, Eng. Educ. Dec. '79, 285 (1979).

${ }^{9}$ J. H. Larkin, Am. J. Phys. 49, 534 (1981).

${ }^{10}$ J. H. Larkin and F. Reif, Eur. J. Sci. Educ. 1, 191 (1979).

"F. Reif and J. I. Heller, Ed. Psychol. 17, 102 (1982).

${ }^{12}$ A. D. de Groot, Thought and Choice in Chess (North-Holland, Amsterdam, 1946).

${ }^{13}$ C. T. C. W. Mettes, A. Pilot, and H. J. Roosink, Instr. Sci. 10, 333 (1981).

${ }^{14}$ D. M. Bunce and H. Heikkinen, J. Res. Sci. Ed. 23, 11 (1986).

${ }^{15}$ A. Arons, Phys. Teach. 19, 166 (1981).

${ }^{16}$ A. Arons, Phys. Teach. 21, 576 (1983).

${ }^{17}$ A. Arons, Phys. Teach. 22, 21 (1984)

${ }^{18}$ A. Arons, Phys. Teach. 22, 88 (1984). 